\title{
Cuestionario de Necesidades de Orientación Universitaria
}

\author{
Questionary of Necessities from University Orientation
}

\author{
Paulina E. Moreno-Yaguana ${ }^{1}$ y Maria Fe Sanchez-Garcia ${ }^{2}$
}

\begin{abstract}
Resumen
Este estudio recoge el diseño de un cuestionario para diagnosticar las necesidades de orientación universitaria en el contexto de Ecuador. Está configurado por dos escalas, la primera sobre las necesidades individuales percibidas y la segunda relativa a los servicios considerados necesarios para los programas de orientación. En una primera fase se analiza la validez de contenido del instrumento a través del juicio de expertos $(n=31)$. En la segunda, se estudian las características psicométricas mediante su aplicación a una muestra de estudiantes universitarios $(\mathrm{n}=1530)$. Los resultados de los análisis factoriales (AFE y AFC) aportan un modelo de tres factores en la primera escala (explicativas del $65.9 \%$ de la varianza) y otro unidimensional en la segunda ( $70.7 \%$ de la varianza). Se comprueba igualmente la consistencia interna $(\alpha=.949$ y .925), concluyendo acerca de la idoneidad del instrumento para identificar las necesidades de orientación académica, personal y profesional de los universitarios ecuatorianos.
\end{abstract}

Palabras clave: necesidades de orientación, orientación universitaria, bienestar, evaluación

\begin{abstract}
This study covers the design of a questionnaire to diagnose the necessities from a university guidance in the context of Ecuador. It is formed by two scales, the first is about the individual necessities perceived, and the second is related to the services considered necessary to guidance programmes. In a first stage, it is analysed the content validity of the tool on the basis of expert opinions $(n=31)$. In the second one, the psychometric characteristics are studied through their application to a sample of university students $(n=1530)$. The factory analysis results (AFE and AFC) contribute a three-factor model in the first scale (explanatories of 65.9\% of the variance) and only one dimension in the second one (70.7\% of the variance). The internal consistency is also tested ( $\alpha=.949$ and .925$)$, concluding about the suitability of the tool for identifying the necessities of academic, personal and professional guidance of Ecuadorian students.
\end{abstract}

Keywords: guidance necessities, university guidance, welfare, evaluation

\footnotetext{
${ }^{1}$ Licenciada en Psicología y Doctoranda de la Universidad Nacional de Educación a Distancia (UNED), España. Universidad Técnica Particular de Loja. Profesora de Universidad. San Cayetano Alto, S.N, Ciudad de Loja, Ecuador. Correo: pemoreno@utpl.edu.ec

${ }^{2}$ Doctora en Ciencias de la Educación, Universidad Nacional de Educación a Distancia (UNED). Profesora Titular de Universidad. Juan del Rosal 14, 28040 Madrid, España. Correo: mfsanchez@edu.uned.es
} 


\section{Introducción}

La incorporación a la universidad constituye una de las transiciones vitales con mayor impacto en el desarrollo profesional, social, personal y emocional de los estudiantes. La orientación universitaria constituye un conjunto planificado de acciones de apoyo proporcionadas desde su propia universidad, para poder afrontar esta transición y para prepararles para gestionar futuras transiciones a lo largo de sus vidas. Como señalaba Rodríguez-Moreno (2002), tanto la variedad del alumnado como los cambios de los sistemas de educación superior, implican la necesidad de orientación e información actualizadas y en un formato que se adapte a sus necesidades concretas.

En contextos internacionales como la conferencia europea de Lisboa de 2000, se reconocía el papel de la relevancia de la orientación para facilitar la transición a la universidad, así como el paso de ésta al mundo laboral. La acción orientadora, en sus diversas modalidades, aparece como algo fundamental en la preparación para un aprendizaje a lo largo de la vida. En el ámbito latinoamericano, se viene insistiendo en su importancia para la adaptación frente a las transformaciones sociopolíticas y económicas que se suceden en el continente, así como en la necesidad de desarrollar y aplicar criterios de coherencia, cooperación y calidad en la orientación (González \& Ledezma, 2009). El I Congreso Iberoamericano de Orientación (U. de La Plata, 2003) recalcaba además su papel para contribuir a reducir las desigualdades sociales, y la necesidad de vincular la praxis orientadora a la propia realidad sociocultural, a la diversidad de perfiles y a su la identidad étnica y cultural (Bonadiman, Scaff, Bardagi, \& Luna, 2015; González- Bello, 2007).

En América Latina se han producido avances significativos respecto a la orientación universitaria (Pezzano de Vengoechea, 2009), y muestra de ello es también la conformación de la Red Latinoamérica de Profesionales de Orientación integrada por un grupo representativo de países, tales como Argentina, Bolivia, Brasil, Chile, Costa Rica, Cuba, El Salvador, Guatemala, Honduras, México, Nicaragua, Panamá, Venezuela, Colombia, Ecuador, Paraguay, Perú,
República Dominicana, Puerto Rico y Uruguay. Existen, sin embargo algunas debilidades que afectan a algunos países latinoamericanos, como la ausencia de políticas públicas en la implementación de los servicios de orientación (González \& Ledesma, 2009; Casas Armengol \& Stojanovic, 2013). Un caso a considerar es el de Ecuador, contexto de investigación en el presente estudio.

En la actualidad, el sistema de educación superior del Ecuador cuenta con una Ley Orgánica de Educación Superior (LOES, 2010), que ha contribuido a que las universidades ecuatorianas mejoren su calidad académica y de gestión, al establecer que Las instituciones de educación superior mantendrán una unidad administrativa de Bienestar Estudiantil destinada a promover la orientación vocacional $y$ profesional, facilitar la obtención de créditos, estímulos, ayudas económicas y becas, y ofrecer los servicios asistenciales que se determinen en las normativas de cada institución (artículo 86). Estos nuevos planteamientos jurídicos exigen a las universidades ecuatorianas la creación de líneas de trabajo sustentadas que coordinen y propongan servicios de orientación universitaria de acuerdo con las necesidades que presenten los estudiantes en el inicio, durante $y$ al final de su vida universitaria.

En Ecuador los servicios de orientación universitaria se regulan desde las Unidades de Bienestar Universitario y cuentan con un marco legal específico que les permite estructurar los servicios desde diversos enfoques y prácticas.

La noción de bienestar universitario ha sido conceptuada desde múltiples planteamientos y acepciones, pero principalmente: (1) entendida como la satisfacción de necesidades y dirigida a una mejora de la calidad de vida en la comunidad universitaria; (2) como gestión de acciones formativas para un desarrollo integral del estudiante; y (3) desde un enfoque administrativo, focalizado en la oferta de servicios asistenciales de orden material como: restaurantes, transportes, becas, residencias etc. (Llinás-González, 2009; Montoya-Vásquez, Urrego-Velásquez, \& PáezZapata, 2014; Puente-Burgos, 2009).

\section{Necesidades de orientación}

Desde los diversos enfoques teóricos, las necesidades humanas son consideradas como 
motor fundamental de la actividad de las personas. La teoría de Maslow (1954) considera la necesidad como una fuerza motivadora para actuar, aportando un sistema jerarquizado representado sobre una pirámide con cinco niveles de necesidades: fisiológicas (en la base), de seguridad, sociales, de reconocimiento y de autorrealización (en la cúspide). Su teoría plantea que sólo cuando las necesidades de nivel inferior son satisfechas, se escala hacia las necesidades de nivel superior, generando la autorrealización a largo plazo. Más tarde, Max-Neef (1994) aportó un modelo de diez necesidades sobre las base de categorías axiológicas (subsistencia, protección, afecto, comprensión, participación, ocio, creación, identidad, libertad y trascendencia) que se combinan con las categorías existenciales del individuo. Este enfoque considera que cualquier necesidad humana fundamental no satisfecha de manera adecuada produce una patología (p. 48) y no concibe la existencia de un orden jerárquico que condicione la prevalencia de una necesidad, si bien reconoce un umbral, por debajo del cual puede ser superior la urgencia por satisfacer una determinada necesidad (p. 79).

En el caso de los estudiantes universitarios, generalmente se encuentran cubiertas las necesidades más básicas (fisiológicas y de seguridad), manteniéndose en mayor o menor medida otro abanico de las necesidades. Su diagnóstico es una actividad que debería llevarse a cabo de forma permanente e institucionalizada por parte de las universidades, con el fin de diseñar e implementar programas de acompañamiento e intervención que contribuyan al éxito académico y al desarrollo integral de los universitarios a lo largo de sus estudios (Flores-Buils, Gil-Beltrán, \& Caballer-Miedes, 2012; Lantarón, 2014; VillarVarela \& Méndez-Lois, 2014). Es preciso, por tanto, implementar modelos de evaluación que permitan a las universidades obtener un grado de conocimiento acerca de la efectividad de los servicios que se ofertan (Vieira Aller, 2008).

Las diversas investigaciones que han girado en torno a las necesidades orientación universitaria coinciden en señalar que los programas y servicios de orientación cumplen diversas funciones focalizadas en orientar e informar sobre un abanico de aspectos académicos, profesionales, de inserción laboral, y de atención a la diversidad (e. g. Farriols Hernando et al., 2014; Guerrero- Romera, 2011; Plant \& Thomsen, 2011; Sánchez-García, 1998; Villena- Martínez, Polo-Sánchez, JiménezRodríguez, \& Muñoz-García, 2010) cumpliendo además la función de prevenir y evitar el abandono universitario (Flores-López, 2015).

Esta preocupación por reducir la deserción universitaria ha propiciado trabajos centrados en identificar factores de éxito académico (e. g. Álvarez- Pérez \& López-Aguilar, 2016; FloresLópez et al., 2015) y también en conocer qué barreras perciben los estudiantes al acceder a la universidad (e. g. Urbanaviciute, Pociute, Kairys, \& Liniauskaite, 2016), aspectos que proporcionan elementos significativos para el estudio de las necesidades de orientación. El diagnóstico de dichas necesidades debe entenderse como un proceso sistemático dirigido a identificar las carencias y dificultades, pero también las potencialidades percibidas, dado que las necesidades patentizan la tensión constante entre carencia y potencia tan propia de los seres humanos (Max-Neef, 1994, p. 49).

Por otro lado, desde el enfoque del desarrollo de la carrera, los resultados de algunos estudios demuestran que la toma de decisiones y la planificación de la carrera son aspectos fundamentales para alcanzar los objetivos personales y profesionales (Betancourt \& Cabrera, 2011; Lee, Rojewski, \& Hill, 2013; Meijers, Kuijpers, \& Gundy, 2013). A su vez, investigaciones como las de Carrasco, Zuñiga y Espinoza (2014), Lerchundi, Núñez del Río y González, (2015) o Liu, McMahon y Watson (2015), demuestran que las aspiraciones académicas y profesionales son dimensiones moldeadas por las estructuras sociales, siendo la familia un soporte importante para el desarrollo personal y profesional.

Desde la perspectiva metodológica son diversos los planteamientos en torno al diagnóstico de las necesidades. Desde un enfoque sistémico, los modelos de Kaufman (1982), de Stufflebeam y Shinkfield (1987) o, más recientemente, de Pérez-Campanero (2010) se plantean el diagnóstico de déficits, para planificar y redefinir las metas de intervención. Para ese proceso, es preciso disponer de técnicas y 
instrumentos que se ajusten a los tipos de necesidades a evaluar y describir.

De esta manera, el estudio en el que se enmarca el presente artículo forma parte de una tesis doctoral cuya finalidad está centrada en valorar la actuación de los servicios de orientación y bienestar universitarios sobre la base del análisis de las necesidades de los estudiantes universitarios ecuatorianos, como un aporte al fortalecimiento y desarrollo de dichos servicios. Para ese propósito, a través del presente trabajo se ha diseñado y validado un cuestionario sobre las necesidades de orientación universitaria en los ámbitos académico, personal y profesional de los estudiantes de Ecuador. Para ello se ha tomado como base el modelo de Sánchez-García (1998), adaptando el Cuestionario sobre necesidades de orientación de los universitarios (CNOU), dado que la distancia temporal y el contexto sociocultural específico del Ecuador aconsejaban una adaptación del instrumento.

Es preciso considerar que la calidad métrica de un cuestionario debe ser evaluada por procedimientos científicos, lo que requiere aplicar índices de fiabilidad y validez (Chávez-Guerrero, Peinado-Pérez, Ornelas-Contreras, \& BlancoVega, 2013; Inzunza-Melo, et al., 2014). Consecuentemente, el objetivo de este trabajo es diseñar y validar un instrumento de diagnóstico válido y fiable sobre las necesidades de orientación de los universitarios ecuatorianos. Específicamente, se pretende: (1) Comprobar la validez de contenido del instrumento; (2) estudiar su validez de constructo, analizando la estructura factorial de sus escalas; y (3) comprobar su consistencia interna.

\section{Método}

\section{Procedimiento}

El proceso de construcción del cuestionario se realizó en dos fases entre 2014 y 2015. En la primera fase, se realizó un estudio piloto sobre la versión adaptada del cuestionario, compuesto por 74 ítems. Primeramente, tras el análisis y revisión de la literatura sobre las necesidades de orientación, se elaboró una primera versión adaptada del CNOU que fue sometida a una evaluación de expertos para verificar la validez de su contenido. En esta fase, la valoración de los expertos se llevó a cabo a través de un protocolo enviado por vía electrónica, a través del cual se les invitó a valorar los criterios de pertinencia (con relación a las distintas dimensiones), suficiencia (si las preguntas son suficientes para abordar la dimensión) y claridad (en la formulación de la pregunta). Se empleó una escala tipo Likert con cinco categorías ( $1=$ mínima adecuación y $5=$ máxima adecuación). Adicionalmente se ofrecieron espacios para expresar abiertamente observaciones y sugerencias generales $\mathrm{o}$ específicas.

En la segunda fase, el instrumento fue aplicado a una muestra de estudiantes ecuatorianos con el fin de analizar la estructura factorial de sus escalas (validez de constructo) y comprobar la fiabilidad del conjunto del instrumento. Como resultado de ambos procesos, se realizó la depuración del instrumento obteniendo la versión final (Apéndice 1).

\section{Instrumento}

El CNOU (Sánchez-García, 1998) fue elaborado y validado en 1997 en el contexto de la población universitaria española y establece un modelo estructurado en necesidades de orientación de tipo académico (asociadas a la adaptación a vida académica y al aprendizaje), profesional (relacionadas con la actual o futura inserción laboral y profesional) y personal (vinculada a otros aspectos que conectan con sus condiciones y circunstancias personales). Si bien su contenido sigue siendo esencialmente relevante para los objetivos de nuestra investigación (Farriols et al., 2014; Figuera \& Coiduras, 2013; Gonzalez-Ramírez, Contreras-Rosado, \& Reyes de Cózar, 2015; Manzano-Soto, \& RoldánMorales, 2015).

El instrumento diseñado para este trabajo, denominado Cuestionario sobre las necesidades de orientación universitaria en Ecuador (CNOU$E)$, en su versión final, se compone de 78 ítems distribuidos en 4 secciones: 1) Datos sociodemográficos; 2) Elección de estudios y orientación; 3) Orientación y bienestar universitario. Se utilizaron opciones de respuesta dicotómicas y escalas tipo Likert de 5 puntos ( $0=$ ninguna necesidad y $5=$ =máxima necesidad $)$.

De los ítems seleccionados, 34 ítems corresponden al instrumento original $\mathrm{CNOU}$ 
Tabla 1. Perfil demográfico de la muestra global

\begin{tabular}{|c|c|c|c|}
\hline \multicolumn{2}{|r|}{ Categorías de análisis } & Frec & DT \\
\hline Sexo & $\begin{array}{l}\text { Mujer } \\
\text { Varón }\end{array}$ & $\begin{array}{l}683 \\
747\end{array}$ & .500 \\
\hline Edad & $\begin{array}{l}17-18 \text { años } \\
19-20 \text { años } \\
21-22 \text { años } \\
23-24 \text { años } \\
25-29 \text { años } \\
30-40 \text { años }\end{array}$ & $\begin{array}{c}73 \\
350 \\
774 \\
147 \\
54 \\
10\end{array}$ & .869 \\
\hline Área disciplinar & $\begin{array}{c}\text { Humanidades y artes } \\
\text { Ciencias sociales, educación, comercial y } \\
\text { derecho } \\
\text { Ciencias } \\
\text { Ingeniería, industria y construcción } \\
\text { Agricultura } \\
\text { Salud y ciencias sociales } \\
\text { Servicios }\end{array}$ & $\begin{array}{c}71 \\
430 \\
376 \\
248 \\
126 \\
109 \\
64\end{array}$ & 1.520 \\
\hline Discapacidad & $\begin{array}{l}\text { Sí } \\
\text { No }\end{array}$ & $\begin{array}{c}52 \\
1372\end{array}$ & .188 \\
\hline $\begin{array}{l}\text { Traslado de } \\
\text { residencia }\end{array}$ & $\begin{array}{l}\text { Sí } \\
\text { No }\end{array}$ & $\begin{array}{l}423 \\
989\end{array}$ & .458 \\
\hline Actividad laboral & $\begin{array}{l}\text { Sí } \\
\text { No }\end{array}$ & $\begin{array}{c}222 \\
1196 \\
\end{array}$ & .399 \\
\hline
\end{tabular}

(Sánchez-García, 1998), cuyo contenido es coherente con los objetivos y el contexto de nuestro estudio. Así mismo, como resultado del estudio piloto y considerando, algunas observaciones y sugerencias relevantes de los expertos, se omitieron algunos ítems, se mejoró la redacción de otros y se incorporaron cuatro nuevos ítems, referidos a: la presencia de discapacidad, la movilidad para estudiar, el abandono de los estudios y la valoración de los servicios que ofrecen las universidades. La versión final del cuestionario fue diseñada en formato online, a través de la herramienta Survey Monkey.

\section{Participantes}

La muestra utilizada se compone de 1430 estudiantes seleccionados de forma incidental, pertenecientes a la Universidad Técnica Particular de Loja (UTPL), Ecuador. La selección de esta Universidad atiende a la circunstancia de que su alumnado cumple con diversos criterios de diversidad dado que (a) se encuentra distribuido en todo el territorio nacional, (b) en un amplio abanico de estudios. Los criterios de selección de la muestra se centraron en asegurar un equilibrio de sexos y de titulaciones cursadas.

La muestra se distribuye en 20 de las 25 provincias ecuatorianas, aunque con mayor presencia en Loja (13.8\%) y Zamora Chinchipe (7.8\%). El 52.2\% de los encuestados fueron hombres y el $47.8 \%$ mujeres $(\mathrm{DT}=.500)$, con edades comprendidas entre los 17 y los 36 años (media de 21.1 años, DT=1.861). Por áreas disciplinares (establecidas por la Secretaría Nacional de Educación Superior, Ciencia, Tecnología e Innovación - SENESCYT), el 30.1 realiza estudios de Ciencias sociales, educación, comercial y derecho, el $26.3 \%$ de Ciencias, el $17.3 \%$ de Ingeniería, industria y construcción, el $8.8 \%$ de Agricultura, el $7.6 \%$ de Salud y servicios sociales, el 5.0\% de Humanidades y artes y el $4,5 \%$ de Servicios. El perfil demográfico de la muestra se recoge en la Tabla 1.

En la validación de contenido del cuestionario participaron 31 expertos seleccionados en función de su conocimiento del ámbito de la orientación educativa y del contexto universitario. De ellos, el $45.3 \%$ son doctores. Respecto a su perfil laboral, el $51.6 \%$ son docentes universitarios (de ellos, 3 
Tabla 2. Análisis comparativo. Equivalencia entre las submuestras utilizadas en los AFE y AFC

\begin{tabular}{lccccc}
\hline & & Muestra para & \multicolumn{2}{c}{ Muestra para } & \multicolumn{2}{c}{ Test de contraste } \\
\multicolumn{2}{c}{ Variables (descriptivos) } & AFE (N=715) & AFC (N=715) & Valor & P-sig \\
\hline Edad & Media (DT) & $21.13(1.84)$ & $21.03(1.89)$ & T=0.93 & .353 \\
\multirow{2}{*}{ Sexo } & $\%$ (frec.) & V: $53.7 \%(384)$ & V: $50.8 \%(363)$ & \multirow{2}{*}{ Chi $^{2}=1.24$} & \multirow{2}{*}{.266} \\
\hline
\end{tabular}

Tabla 3. Valoración de expertos. Pertinencia, suficiencia y claridad

\begin{tabular}{lcccccc}
\hline \multirow{2}{*}{ Dimensiones } & \multicolumn{2}{c}{ Pertinencia } & \multicolumn{2}{c}{ Suficiencia } & \multicolumn{2}{c}{ Claridad } \\
& $\mathrm{M}$ & $\mathrm{DT}$ & $\mathrm{M}$ & $\mathrm{DT}$ & $\mathrm{M}$ & $\mathrm{DT}$ \\
\hline 1.1. Sexo & 4.90 & 152.00 & 4,84 & 150.00 & 4.90 & 152.00 \\
1.2. Edad & 5.00 & 155.00 & 4,84 & 150.00 & 4.87 & 151.00 \\
1.3. Estado Civil & 4.84 & 150.00 & 4,65 & 144.00 & 4.84 & 150.00 \\
1.4. Carrera y Ciclo & 4.65 & 144.00 & 4,55 & 141.00 & 4.35 & 135.00 \\
1.5 Discapacidad & 4.84 & 150.00 & 4,35 & 135.00 & 4.42 & 137.00 \\
1.6 Desplazamiento & 4.84 & 150.00 & 4,55 & 141.00 & 4.06 & 126.00 \\
$\begin{array}{l}\text { 1.7 Cargas laborales } \\
\text { 2. Escala NO (necesidades de }\end{array}$ & 4.77 & 148.00 & 4,13 & 128.00 & 4.03 & 125.00 \\
orientación) & 4.84 & 150.00 & 4,48 & 139.00 & 4.65 & 144.00 \\
3. Escala SN (servicios & & & & & & \\
necesarios) & 4.52 & 140.00 & 4,42 & 137.00 & 4.29 & 133.00 \\
4. Sugerencias para los & & & & & & \\
servicios de orientación & 5.00 & 155.00 & 4,90 & 152.00 & 4.77 & 148.00 \\
\hline
\end{tabular}

Nota: $\mathrm{M}=$ Media; DT= Desviación típica

eran decanos de facultades de psicología), el $16.1 \%$ directores de servicios de bienestar universitario, el $16.1 \%$ trabajadores(as) sociales y un $16.1 \%$ psicólogos(as) de los servicios universitarios. El $58.1 \%$ de los expertos pertenecen a universidades privadas, mientras que el $41.9 \%$ a universidades públicas. Geográficamente, el $64.5 \%$ procede de universidades ecuatorianas, mientras que el $35.5 \%$ están adscritos a universidades extranjeras (España, Estados Unidos y Chile).

\section{Análisis de datos}

Para el estudio factorial, al disponer de este elevado $\mathrm{N}$ de casos, se procede a realizar una división de la muestra en dos submuestras por el procedimiento del muestreo aleatorio simple, de igual tamaño $(\mathrm{N}=715)$ y equivalentes en edad y sexo. La Tabla 2 muestra esta equivalencia, al no existir diferencias estadísticamente significativas $(p>.05)$. Tampoco se encontraron diferencias significativas $(p>.05)$ en estado civil, carrera que cursa y nivel/ciclo de estudios.

Con el fin de dotar de mayor potencia al análisis estadístico de la validación, a continuación se procedió a utilizar la primera submuestra para la realización del análisis factorial exploratorio (AFE) y la segunda para el análisis factorial confirmatorio (AFC).

En el estudio de la fiabilidad, tanto para la versión piloto como para la definitiva, se aplicó el índice Alpha de Cronbach. La estructura factorial del cuestionario final (validez de constructo) se exploró a través del análisis factorial (método de componentes principales, con rotación Varimax).

Para este análisis comparativo de submuestras, los AFE y análisis de fiabilidad se utilizó el paquete estadístico IBM SPSS 22; para el AFE, la aplicación AMOS 22.0 asociada a SPSS.

\section{Resultados}

\section{Valoración de expertos}

Atendiendo al objetivo específico 1, los resultados sobre la valoración global del cuestionario por parte de los expertos ofrecieron valores medios elevados en todos los criterios, por encima de 4 puntos (en la escala de 1 a 5): suficiencia $(\mathrm{M}=4.06, \mathrm{DT}=0.679)$, pertinencia $(\mathrm{M}=4.52, \quad \mathrm{DT}=0.676)$ y claridad $(\mathrm{M}=4.35$, $\mathrm{DT}=0.709)$. En la Tabla 3 se recogen los resultados 
Tabla 4. Análisis factorial exploratorio. Escala NO - Necesidades de orientación

\begin{tabular}{|c|c|c|c|c|c|c|c|}
\hline \multirow{2}{*}{$\mathrm{N}^{\mathrm{o}}$ Ítem } & \multicolumn{3}{|c|}{ Descriptivos $(\mathrm{n}=714)$} & \multicolumn{4}{|c|}{ Saturaciones $>.500$} \\
\hline & Media & $\mathrm{DE}$ & Comuna-lidad & Factor 1 & & & Factor 3 \\
\hline $18 \mathrm{u}$ & 2.31 & 1.89 & .688 & .918 & & & \\
\hline $18 \mathrm{v}$ & 2.42 & 1.79 & .705 & .888 & & & \\
\hline $18 \mathrm{t}$ & 2.56 & 1.76 & .741 & .879 & & & \\
\hline $18 \mathrm{~s}$ & 2.59 & 1.72 & .734 & .841 & & & \\
\hline $18 \mathrm{r}$ & 2.79 & 1.69 & .674 & .752 & & & \\
\hline $18 \mathrm{q}$ & 2.98 & 1.60 & .655 & .686 & & & \\
\hline 180 & 2.65 & 1.84 & .515 & .622 & & & \\
\hline $18 \mathrm{p}$ & 2.95 & 1.59 & .680 & .606 & & & \\
\hline $18 \mathrm{~m}$ & 3.52 & 1.48 & .830 & & & & \\
\hline 181 & 3.47 & 1.47 & .796 & & & & \\
\hline $18 \mathrm{k}$ & 3.46 & 1.52 & .760 & & & & \\
\hline $18 n$ & 3.49 & 1.51 & .753 & & & & \\
\hline $18 \mathrm{j}$ & 3.16 & 1.58 & .540 & & & & \\
\hline $18 \mathrm{i}$ & 3.47 & 1.47 & .796 & & & & \\
\hline $18 \mathrm{a}$ & 2.73 & 1.73 & .602 & & & & .879 \\
\hline $18 \mathrm{~b}$ & 3.01 & 1.51 & .649 & & & & .831 \\
\hline $18 \mathrm{e}$ & 2.75 & 1.62 & .648 & & & & .733 \\
\hline $18 \mathrm{~d}$ & 3.14 & 1.49 & .667 & & & & .697 \\
\hline $18 \mathrm{c}$ & 3.00 & 1.50 & .580 & & & & .682 \\
\hline $18 \mathrm{f}$ & 3.00 & 1.61 & .606 & & & & .671 \\
\hline $18 \mathrm{~g}$ & 2.75 & 1.72 & .535 & & & & .509 \\
\hline & Correlaci & tre los & & $\mathrm{F} 1$ & -- & 603 & .582 \\
\hline & & & & $\mathrm{F} 2$ & & -- & .685 \\
\hline
\end{tabular}

específicos de cada sección. Puede observarse que se obtienen valores promedio igualmente elevados. Cabe reseñar que un ítem de la sección de datos sociodemográficos, referido a la identidad cultural, recibió valoraciones discrepantes y comentarios de varios expertos cuestionando su idoneidad al considerar la compleja diversidad de mestizaje existente en el Ecuador y la consiguiente dificultad para establecer opciones claras y excluyentes respecto al origen cultural. En consecuencia, se decidió omitir este ítem en la versión final del cuestionario. Asimismo, se revisó la redacción de algunos ítems que recibieron menor valoración promedio en el criterio de claridad o comentarios por parte de los jueces.

\section{Análisis factorial}

Para el estudio factorial de las dos escalas del cuestionario (objetivo específico 2), se ha aplicado primeramente un análisis factorial exploratorio (AFE), seguido de un análisis factorial confirmatorio (AFC). Previamente se comprobó la idoneidad del análisis factorial para la distribución de datos mediante la obtención del índice Kaiser-Meyer-Olkin.

En el caso de la Escala NO (Necesidades de orientación), compuesta por 21 ítems en escala Likert de 6 puntos $(0=$ ninguna necesidad $/ 5=$ máxima necesidad), se aprecia en primer lugar que la ratio sujetos/ítem es $34.05(715 / 21)$ y por tanto supera el mínimo necesario. Se verifican correctamente las condiciones previas necesarias para la realización de un AFE: (1) índice KMO muy elevado (.945); (2) significatividad en el Test de Esfericidad rechazando la hipótesis de matriz identidad ( $p<.000001) ;$ y (3) valor del determinante de la matriz (0.000) prácticamente nulo en la línea de lo anterior.

Para la extracción de los factores se empleó el método de Componentes Principales con rotación a través del método oblicuo Promax, cuyos resultados se recogen en la Tabla 4. Los ítems presentan muy buenas comunalidades, articulados en una estructura con 3 factores correlacionados entre sí, que explican el $65.95 \%$ de la variabilidad total. El primero de estos factores ya explica un $51.04 \%$ de ese total, en tanto que los otros dos añaden porcentajes sensiblemente menores, en concreto un $9.52 \%$ el factor 2 y un $5.39 \%$ el factor 3 . 


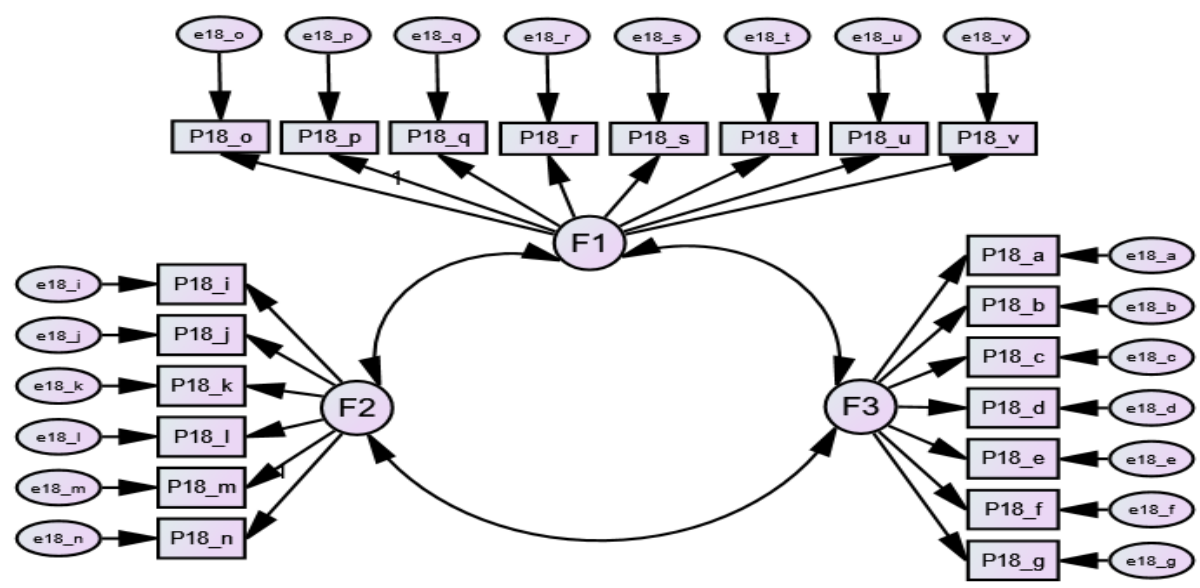

Figura 1. Diagrama de flujo del AFC. Escala NO

Tabla 5. Análisis Factorial Confirmatorio. Escala NO

\begin{tabular}{|c|c|c|c|c|c|}
\hline Modelo & & RMSEA & $\mathrm{NF}$ & IFI & CFI \\
\hline Tres factores & & .127 & .77 & .785 & .785 \\
\hline \multirow{2}{*}{$\mathrm{N}^{\mathrm{o}}$ Ítem } & \multicolumn{4}{|c|}{ Coef. de regresión estandarizados } & \multirow{2}{*}{ Ecuación estructural } \\
\hline & & Factor 1 & Factor 2 & Factor 3 & \\
\hline $18-\mathrm{t}$ & & .866 & & & $\mathrm{~F} 1=2.87+1.64 \times \mathrm{P} 18-\mathrm{t}$ \\
\hline $18-\mathrm{s}$ & & .841 & & & $\mathrm{~F} 1=2.63+1.57 \times \mathrm{P} 18-\mathrm{s}$ \\
\hline $18-\mathrm{v}$ & & .839 & & & $\mathrm{~F} 2=2.46+1.65 \times \mathrm{P} 18-\mathrm{v}$ \\
\hline $18-\mathrm{u}$ & & .810 & & & $\mathrm{~F} 1=2.41+1.67 \times \mathrm{P} 18-\mathrm{u}$ \\
\hline $18-\mathrm{r}$ & & .801 & & & $\mathrm{~F} 1=2.87+1.43 \times \mathrm{P} 18-\mathrm{r}$ \\
\hline $18-q$ & & .776 & & & $\mathrm{~F} 1=2.98+1.37 \times \mathrm{P} 18-\mathrm{q}$ \\
\hline $18-\mathrm{p}$ & & .768 & & & $\mathrm{~F} 1=2.98+1.33 \times \mathrm{P} 18-\mathrm{p}$ \\
\hline $18-0$ & & .377 & & & $\mathrm{~F} 1=2.59+1.00 \times \mathrm{P} 18-\mathrm{o}$ \\
\hline $18-1$ & & & .860 & & $\mathrm{~F} 2=3.61+1.15 \times \mathrm{P} 18-1$ \\
\hline $18-\mathrm{m}$ & & & .855 & & $\mathrm{~F} 2=3.52+1.21 \times \mathrm{P} 18-\mathrm{m}$ \\
\hline $18-\mathrm{k}$ & & & .822 & & $\mathrm{~F} 2=3.60+1.16 \times \mathrm{P} 18-\mathrm{k}$ \\
\hline $18-n$ & & & .757 & & $\mathrm{~F} 2=3.59+1.00 \times \mathrm{P} 18-\mathrm{n}$ \\
\hline $18-\mathrm{j}$ & & & .605 & & $\mathrm{~F} 2=3.23+0.96 \times \mathrm{P} 18-\mathrm{j}$ \\
\hline $18-\mathrm{i}$ & & & .604 & & $\mathrm{~F} 2=3.18+1.06 \times \mathrm{P} 18-\mathrm{i}$ \\
\hline $18-d$ & & & & .835 & $\mathrm{~F} 3=3.16+1.32 \times \mathrm{P} 18-\mathrm{d}$ \\
\hline $18-\mathrm{c}$ & & & & .760 & $\mathrm{~F} 3=3.10+1.19 \times \mathrm{P} 18-\mathrm{c}$ \\
\hline $18-\mathrm{f}$ & & & & .760 & $\mathrm{~F} 3=3.03+1.23 \times \mathrm{P} 18-\mathrm{f}$ \\
\hline $18-b$ & & & & .745 & $\mathrm{~F} 3=3.10+1.17 \times \mathrm{P} 18-\mathrm{b}$ \\
\hline $18-e$ & & & & .732 & $\mathrm{~F} 3=2.85+1.21 \times \mathrm{P} 18-\mathrm{e}$ \\
\hline $18-\mathrm{a}$ & & & & .713 & $\mathrm{~F} 3=2.93+1.21 \times \mathrm{P} 18-\mathrm{h}$ \\
\hline $18-\mathrm{g}$ & & & & .696 & $\mathrm{~F} 3=2.86+1.18 \times \mathrm{P} 18-\mathrm{g}$ \\
\hline Correlación entre & $\mathrm{F} 1$ & -- & .602 & .684 & \\
\hline factores & $\mathrm{F} 2$ & & -- & .741 & \\
\hline
\end{tabular}

El análisis factorial confirmatorio (AFC) permite comprobar el modelo de factores obtenido, representado en la Figura 1. Se trata de una estructura de 3 factores correlacionados entre sí, y con un número de ítems similar unos con respecto a los otros $(8,6$ y 7$)$. El modelo obtenido es coherente con la configuración teórica aplicada en el diseño. El primer factor, que se ha denominado necesidades de orientación personal, agrupa 8 ítems sobre aspectos de bienestar más holísticos o complementarios al desarrollo académico; el segundo factor, necesidades de orientación profesional, se compone de 6 ítems asociados al futuro profesional; y el tercer factor, denominado necesidades de orientación académica, está configurado por otros 7 ítems relacionados con aspectos de la vida universitaria. 
Tabla 6. Análisis Factorial Exploratorio y Confirmatorio. Escala SN

\begin{tabular}{|c|c|c|c|c|c|}
\hline \multirow{2}{*}{$\mathrm{N}^{\mathrm{o}}$ Ítem } & \multicolumn{3}{|c|}{ Descriptivos } & \multirow{2}{*}{\multicolumn{2}{|c|}{$\begin{array}{c}\text { Saturaciones }>.500 \\
\text { Factor } 1\end{array}$}} \\
\hline & Media & D.E. & Comunalidad & & \\
\hline $19-\mathrm{c}$ & 3.43 & 1.59 & .755 & \multicolumn{2}{|c|}{.869} \\
\hline $19-\mathrm{f}$ & 3.57 & 1.64 & .751 & \multicolumn{2}{|c|}{.867} \\
\hline $19-d$ & 3.66 & 1.55 & .743 & \multicolumn{2}{|c|}{.862} \\
\hline 19-b & .329 & 1.62 & .724 & \multicolumn{2}{|c|}{.851} \\
\hline $19-\mathrm{g}$ & 3.65 & 1.65 & .702 & \multicolumn{2}{|c|}{.838} \\
\hline $19-\mathrm{e}$ & 3.74 & 1.58 & .674 & \multicolumn{2}{|c|}{.821} \\
\hline $19-\mathrm{a}$ & 3.09 & 1.82 & .600 & \multicolumn{2}{|c|}{.775} \\
\hline Modelo & \multicolumn{2}{|l|}{ RMSEA } & IFI & TLI & $\mathrm{CFI}$ \\
\hline Un factor & .186 & .899 & .902 & .853 & .902 \\
\hline $\mathrm{N}^{o}$ Ítem & \multicolumn{3}{|c|}{ Coef. de regresión estandarizados } & \multicolumn{2}{|c|}{ Ecuación estructural } \\
\hline $19-d$ & \multicolumn{3}{|c|}{.859} & \multicolumn{2}{|c|}{$\mathrm{F} 1=3.80+1.22 \times \mathrm{P} 13-\mathrm{d}$} \\
\hline $19-\mathrm{c}$ & \multicolumn{3}{|c|}{.853} & \multicolumn{2}{|c|}{$\mathrm{F} 1=3.49+1.30 \times \mathrm{P} 13-\mathrm{c}$} \\
\hline 19-b & \multicolumn{3}{|c|}{.814} & \multicolumn{2}{|c|}{$\mathrm{F} 1=3.36+1.28 \times \mathrm{P} 13-\mathrm{b}$} \\
\hline 19-f & \multicolumn{3}{|c|}{.808} & \multicolumn{2}{|c|}{$\mathrm{F} 1=3.63+1.25 \times \mathrm{P} 13-\mathrm{f}$} \\
\hline $19-\mathrm{g}$ & \multicolumn{3}{|c|}{.755} & \multicolumn{2}{|c|}{$\mathrm{F} 1=3.69+1.17 \times \mathrm{P} 13-\mathrm{g}$} \\
\hline $19-\mathrm{e}$ & \multicolumn{3}{|c|}{.750} & \multicolumn{2}{|c|}{$\mathrm{F} 1=3.81+1.12 \times \mathrm{P} 13-\mathrm{e}$} \\
\hline $19-\mathrm{a}$ & \multicolumn{3}{|c|}{.693} & \multicolumn{2}{|c|}{$\mathrm{F} 1=3.18+1.23 \times \mathrm{P} 13-\mathrm{a}$} \\
\hline
\end{tabular}

Como puede observarse en la Tabla 5, RMSEA no se encuentra por debajo de corte y su valor superior se sitúa en el IC (95\%): .122-.132; así mismo los valores del resto de los índices no alcanzan el punto de corte (.800) aunque se sitúan muy cerca del mismo. En consecuencia, el ajuste es aceptable, y permite validar el resultado del AFC. Los coeficientes estandarizados son altamente significativos $(p<.0001)$ comprobando así la certeza de la asociación de los ítems con su correspondiente factor esperado según el modelo. Solamente el ítem 18-o manifiesta una asociación algo más débil de la esperada con respecto al F1; pero podría ser mantenido dentro de la estructura. Por otro lado el resultado obtenido también prueba la existencia de elevadas correlaciones entre las 3 dimensiones. Globalmente, por tanto, se verifica un correcto ajuste al modelo detectado a través del AFE.

Respecto a la Escala SN (Servicios necesarios), se redactaron 7 ítems, igualmente en escala Likert de 6 puntos $(0=$ ninguna necesidad $/$ 5 = máxima necesidad). La ratio sujetos/ítem es $10.7(715 / 7)$ es igualmente muy superior al mínimo necesario, cumpliéndose sobradamente las condiciones previas necesarias para la realización de un AFE: (1) el índice KMO es elevado (.916); (2) hay significatividad en el Test de Esfericidad de Bartlett (valor Chi-cuadrado: $3985.69 ; 21 \mathrm{gl})$ que rechaza la hipótesis de matriz identidad $(p<.0001)$ es decir que los ítems intercorrelacionan; y (3) el determinante de la matriz (0.004) confirma esta cuestión. Para el AFE se ha utilizado igualmente el método de extracción de Componentes Principales con rotación Promax.

Según puede apreciarse en la Tabla 6, la totalidad de los ítems presentan altas comunalidades. Los diversos métodos de extracción probados coinciden en detectar una composición unidimensional para este conjunto de ítems, explicando un elevado porcentaje de la varianza total: $70.71 \%$, y siendo los pesos factoriales muy elevados.

En la Tabla 6 se presentan también los coeficientes estandarizados del AFC, junto con los valores estadísticos de ajuste. En este caso, el valor de RMSEA se aleja del punto corte adecuado, siendo su valor uno de los comprendidos en el IC (95\%): .170 - .203. Si bien esto podría sembrar dudas acerca del ajuste de los datos, sin embargo, los restantes índices superan el corte (.800) muy claramente. Por tanto, cabe admitir la validez de AFC con un ajuste más que 
Tabla 7. Análisis de fiabilidad. Escalas NO y SN

\begin{tabular}{lcccc}
\hline Factor & $N^{\circ}$ ítems & $\alpha$ Cronbach & IC 95\% & Test de significación \\
\hline ESCALA NO & 21 & .949 & $.945-.953$ & $<.000$ \\
F1-Or.personal & 8 & .920 & $.914-.926$ & $<.000$ \\
F2-Or.profesional & 6 & .896 & $.888-.904$ & $<.000$ \\
F3-Or.académica & 7 & .889 & $.880-.898$ & $<.000$ \\
ESCALA SN & 7 & .925 & $.918-.930$ & $<.000$ \\
\hline
\end{tabular}

aceptable. Vemos que los coeficientes estandarizados son altamente significativos $(p<.0001)$ verificando con seguridad la asociación de los ítems en la única dimensión del modelo planteado.

\section{Fiabilidad}

Según se aprecia en la Tabla 7, el coeficiente de fiabilidad de la escala NO completa (21 ítems) es muy elevado: .949 (IC al 95\%: .945 - .953). Analizando por factores, el $\mathrm{F} 1$ presenta un coeficiente "alfa" de .920 (IC: .914 - .926); el F2 de .896 (IC: .888 - .904); y finalmente el F3 un coeficiente de .889 (IC: .880 - .898). En consecuencia la fiabilidad de la escala completa y de cada una de sus dimensiones por separado, es elevada.

Finalmente, en la escala unidimensional SN (7 ítems), el coeficiente de Cronbach presenta una vez más un valor muy elevado: .923 (IC: .919 .930 , al 95\%).

\section{Discusión}

Respecto al primer objetivo del estudio, las valoraciones medias que otorgan los expertos sobre los conjuntos de ítems son bastantes satisfactorias, pudiendo concluir que el cuestionario resulta pertinente, claro, y sus ítems son suficientes para el objetivo propuesto, obteniéndose valores entre 4.03 y 5 .

Atendiendo al segundo objetivo, el estudio de la validez de constructo de las escalas ha permitido identificar un modelo unidimensional en la escala de servicios necesarios ( $\mathrm{SN}$ ) y un modelo de tres factores en la escala de necesidades de orientación (NO), configurado por las necesidades de orientación personal (factor 2) que agrupa aspectos de bienestar de carácter más general; las necesidades de orientación profesional (factor 2) compuesto por necesidades vinculadas al futuro desarrollo profesional; y las necesidades de orientación académica (factor 3) vinculado al aprendizaje y la vida universitaria. En conclusión, el AFC confirma la estructura del modelo obtenido a través del AFE.

Respecto al tercer objetivo, se comprueba la alta consistencia interna de ambas escalas $(\alpha=.949$ y .925) y de sus factores. Esto permite concluir acerca de la idoneidad del instrumento para identificar las necesidades de orientación académica, personal y profesional de los universitarios en el Ecuador.

En este sentido, diversos trabajos realizados en el campo de la orientación universitaria coinciden en la importancia de diseñar instrumentos adaptados a las características de la población objeto de estudio, con la finalidad de identificar las necesidades y las opiniones de los estudiantes sobre los servicios de orientación (Creed \& Hood, 2015; García, Conejero, \& Díez, 2014; Litoiu, 2009; Palade \& Constantin, 2012).

Respecto a las dimensiones de necesidad encontradas, los resultados hallados en el contexto universitario portugués sobre necesidades psicológicas por Pinto, Faria, Pinto y Taveira (2016), aunque con matices, identifican necesidades que se enmarcarían en los factores de nuestro estudio: manejo de la ansiedad y miedo al fracaso (en el factor 1), estrategias de búsqueda de empleo (en el factor 2) y elección de una carrera y habilidades de gestión del tiempo (en el factor 3). En el contexto de Colombia, Manzano-Soto y Roldán-Morales (2015) analizaron las necesidades de orientación en estudiantes universitarios de primer curso, definiendo de forma similar perfiles de necesidad asociados a: los aspectos académicos (que estarían vinculados factor 3 de nuestro estudio), los aspectos psicosociales (factor 1) y en relación con su proyecto de vida (factor 2). Estas coincidencias animan a plantear, en futuros estudios, en qué medida este modelo de necesidades está presente en otros contextos nacionales. Sería conveniente corroborar el 
modelo factorial a través de estudios con muestras más plurales respecto a las universidades ecuatorianas de procedencia, así como de otros países del entorno latinoamericano. E igualmente el uso de la herramienta para analizar perfiles de necesidades en función de diversas características de los estudiantes, con el fin de facilitar la planificación coherente de las acciones de orientación.

Es preciso tener presentes, no obstante, las limitaciones del estudio, entre las que cabe considerar la procedencia de la muestra que, si bien garantiza una diversidad de condiciones sociodemográficas, pertenece a un mismo establecimiento universitario. Además de confirmar la estructura factorial a través de nuevos estudios con muestras de diversas universidades, es prudente y aconsejable, en particular, seguir analizando la consistencia interna de la subescala de necesidades de orientación académica.

En definitiva, cabe señalar que la aportación de esta herramienta metodológica facilitará el diagnóstico de las necesidades de índole personal, profesional y académica en la población de estudiantes universitarios ecuatorianos, contribuyendo a adaptar y mejorar la calidad de la praxis orientadora destinada a la formación integral del estudiante. Desde esa perspectiva, y de acuerdo con las apreciaciones de otros autores (e. g. Bonadiman, Scaff, Bardagi, \& Luna, 2015; Farriols Hernando et al., 2014; Guerrero-Romera, 2011; Plant, \& Thomsen, 2011; Sánchez-García, 1998; Villena-Martínez, Polo-Sánchez, JiménezRodríguez, \& Muñoz-García, 2010; Vieira-Aller, 2008), los servicios de orientación universitarios deben sustentar sus intervenciones sobre la focalización de la diversidad y la especificidad de necesidades de sus estudiantes. De acuerdo con Núñez-Rojas, Tobón-Tobón, Arias-Henao y Bañol-Ramírez (2006), esta será una premisa necesaria para alcanzar sistemas articulados de orientación y bienestar universitario que funcionen bajo una lógica de calidad.

\section{Referencias}

Álvarez-Pérez, P. R., \& López-Aguilar, D. (2016). El profesorado y la valoración de las competencias del alumnado universitario de nuevo ingreso. International Conference IAEVG. Promoting Equity through Guidance: Reflection Action Impact. Madrid, 15-18 november.

Bethencourt J. T., \& Cabrera L. (2011). Personalidad y toma de decisiones vocacionales en universitarios. Revista Electrónica de Investigación y Evaluación Educativa, 17(1), 1-14.

Bonadiman, M. D., Scaff, L. A., Bardagi, M.P., \& Luna, I.N. (2015). Perfil dos usuarios do LIOP - Laboratório de Informação Proffissional da UFSC: Mudanças observadas nos últimos anos. Revista de Extensão do IFSC, 1(3), 91-100.

Carrasco E., Zúñiga C., \& Espinoza J. (2014). Elección de carrera en estudiantes de nivel socioeconómico bajo de universidades chilenas altamente selectivas. Calidad en la Educación, 40, 95-128. doi: 10.4067/S071845652014000100004

Casas-Armengol, M., \& Stojanovic, L. (2013). Innovación en la universidad iberoamericana. Revista de Universidad y Sociedad del Conocimiento, 10(1), 61-74. doi: 10.7238/rusc.v10i1.1345

Creed, P., \& Hood, M. (2015). The relationship btween career adaptabilty, person and situation variables, and career concerns in Young adults. Journal of Vocational Behavior, 74(2), 210-229. doi: 10.1016/j.jvb.2008.12.004

Chávez-Guerrero, A., Peinado-Pérez, J., OrnelasContreras, M., \& Blanco-Vega, H. (2013). Composición factorial de una escala de autoeficacia en conductas académicas en universitarios de ingeniería. Revista Iberoamericana de Diagnóstico y Evaluación - e Avaliação Psicológica, 35(1), 77-93.

Farriols-Hernando, N., Vilaregut-Puigdesens, A., Palma-Sevillano, C., Dotras-Ruscalleda, P., Llinares-Fité, M., \& Dalmau-Jordá, C. (2014). Estudio descriptivo de un modelo de orientación universitario. Revista Española de Orientación y Psicopedagogía, 25(3), 106120. doi: 10.5944/reop.vol.25.num.3.2014.13 861

Figuera-Gazo, P., \& Coiduras-Rodríguez, J. L. (2013). La transición a la universidad: Un análisis desde la diversidad de las voces de los 
estudiantes. Revista de Educación, 362, 713736. doi: 10.4438/1988-592X-RE-2013-362247

Flores-Buils, R., Gil-Beltrán, J. M., \& CaballerMiedes (2012). El rol del servicio de orientación en universidades de Francia, EEUU, Canadá, Reino Unido y España. Papeles del Psicólogo, 33(2), 138-147.

Flores-López, W. O. et al. (2015). Centros de Apoyo y Desarrollo Educativo Profesional para la observación y disminución de la deserción universitaria. Ciencia $e$ Interculturalidad, 18(1), 48-62.

García, E., Conejero, J. A., \& Díez, J. L. (2014). La entrada en la universidad: Un reto para la orientación académica. Revista de Docencia Universitaria, 12(2), 255-280.

González, J., \& Ledezma, M A. (2009). La orientación en América Latina: Consideraciones generales acerca de los criterios de coherencia, cooperación y calidad. Orientación y sociedad, 9, 00. Recuperado de http://sedici.unlp.edu.ar/handle/10915/13898

González-Bello, J. R. (2008). La Orientación Profesional en América Latina. Fortalezas, Debilidades, Amenazas y Oportunidades. Revista Mexicana de Orientación Educativa, 13, 44-49.

González-Ramírez, T., Contreras-Rosado, J., \& Reyes de Cózar, S. (2015). Utilización y valoración de los servicios de orientación desde la perspectiva de los universitarios. Investigar con y para la sociedad, 1, 291-303.

Guerrero-Romera, C. (2011). Los servicios de orientación de la enseñanza superior en Europa: Un estudio comparado. Revista Española de Educación Comparada, 17, 219238. doi: 10.5944/reec.17.2011

Inzunza-Melo, B. et al. (2014). Estructura factorial y confiabilidad del cuestionario de satisfacción académica en estudiantes de medicina chilenos. Revista Iberoamericana de Diagnóstico y Evaluación e - Avaliação Psicológica, 40(2), 73-82.

Kaufman, R. (1982). Identyfing and solving problems: A sistem aproach. San Diego, California: University Associates.

Lantarón, B. S. (2014). Los servicios de orientación profesional en las universidades españolas. Revista de Investigación en Educación, 12(1), 73-91.

Ley N. 298 (2010). Ley Orgánica de Educación Superior. Publicada en Registro Oficial Órgano del Gobierno del Ecuador, del 12 de Octubre.

Lee, I. H., Rojewski, J. W., \& Hill, R. B. (2013). Classifying Korean adolescents' career preparedness. International Journal for Educational and Vocational Guidance, 13, 25-45. doi: 10. 1007/s10775-012-9236-5

Lerchundi, I., Nuñez del Río, M., \& González, R. (2015). Career choice in engin students. Anales de Psicología, 31(1), 269-279. doi:10.6018/analesps.31.1.159161

Litoiu, N. (2009). Career Counseling Challlenges in Romanian Universities. Case Study on University Politehnica of Bucharest. Buletinul Universitatii Petrol-Gaze din Ploiesti, 68 (2), 137-142.

Liu, J., McMahon, M., \& Watson, M. (2015), Parental influence on child career development in mainland China: A qualitative study. The Career Development Quarterly, 63(1) ,74-87. doi: 10.1007/s10775-015-92919.

Llinás-González, E. (2009). La Orientación Académica desde el Bienestar Universitario. Barranquilla, Colombia: Uninorte.

Maslow, A. (1954). Motivación y personalidad. Madrid, España: Diaz de Santos.

Manzano-Soto, N. \& Roldán-Morales, C.A. (2015). Análisis de necesidades de orientación del estudiante de primer año en la Universidad Autónoma de Occidente. Revista Española de Orientación y Psicopedagogía, 26(3), 121140. doi: 10.5944/reop.vol.26.num.3.2015.164 04

Max-Neef. M. A. (1994). Desarrollo a escala humana. Conceptos, aplicaciones y algunas reflexiones. Barcelona: Icaria.

Meijers, F., Kuijpers, M., \& Gundy, C. (2013). The relation between career competencies, career identity, motivation and quality of choice. International Journal for Educational and Vocational Guidance, 13(1), 47-66. doi: 10.1007/s10775-012-9237-4.

Montoya-Vásquez, D., Urrego-Velásquez, D., \& Páez-Zapata, E. (2014). Experiencia en la coordinación de programas de bienestar 
universitario: La tensión entre el asistencialismo y el desarrollo humano de los estudiantes. Revista Facultad Nacional de Salud Pública, 32(3),355-363.

Núñez-Rojas, A., Tobón-Tobón, S., Arias Henao, D., \& Bañol Ramírez, J. (2006). Sistema articulado de bienestar universitario. Revisa Hacia la Promoción de la Salud, 11, 61-72.

Palade, A., \& Constantin, C. (2012). The necessity of Counseling and vocational orientation in students career management. Bulletin of the Transilvania University of Brasov, 5(2), 61-68.

Perez-Campanero, M. P. (2010). Como detectar las necesidades de intervención socioeducativa. Madrid: Narcea.

Pérez, J., \& Martínez, M. (2015). Tutoría universitaria y servicios de orientación: Dos realidades en un mismo contexto. Revista Electrónica Interuniversitaria de Formación del Profesorado, 18 (2), 177-192. doi: 10.6018/reifop.18.2.219461

Pezzano de Vengoechea, G. (2009). El contexto de la Orientación Académica desde el Bienestar Universitario. En E. C. LlinásGonzález, La orientación académica desde el bienestar universitario (pp. 1-19). Bogotá: Ediciones Uninorte.

Pinto, J. C., Faria, L., Pinto, H. R., \& Taveira, M. D. C. (2016). Identificação de necessidades de intervenção psicológica: Um estudo-piloto no ensino superior português. Psicologia USP, 27(3). doi: 10.1590/0103-656420150015

Plant, P. \& Thomsen, R (2011). Orientación profesional en Dinamarca: El control social con guantes de seda. Orientación y Sociedad, 11, 2-16.

Puente-Burgos, C. (2009). Medio Universitario y Bienestar Universitario en la Pontificia Universidad Javeriana-Reflexiones en torno a los conceptos. (CENDEX Documento de trabajo 018-09). Recuperado de: http://www.javeriana.edu.co/cendex/GPGD/in formes/InformeBienestarPUJ.pdf

Rodríguez-Moreno, M. L. (2002). Hacia una nueva orientación universitaria. Modelos integrados de acción tutorial, orientación curricular y construcción del proyecto profesional. Barcelona, España: Universitat de Barcelona.
Sánchez-García, M. F. (1998). Las funciones y necesidades de orientación en la universidad: Un estudio comparativo sobre las opiniones de universitarios y profesionales. Revista de Orientación y Psicopedagogía, 9 (15), 87107.

Stufflebeam, D. L., \& Shinkfield, A. J. (1987). Evaluación sistemática. Guía teórica y práctica. Madrid: Paidos, MEC.

Urbanaviciute, I., Pociute, B., Kairys, A., \& Liniauskaite, A. (2016). Perceived career barriers and vocational outcomes among university undergraduates: Exploring mediation and moderation effects. Journal of Vocational Behavior, 92, 12-21. doi: 10.1016/j.jvb.2015.11.001

Vieira-Aller, M.J. (2008). Criterios para la evaluación del sistema de apoyo y orientación al estudiante universitario: Revisión y propuesta. Revista de Educación, 345, 399423.

Villena, M. D., Muñoz, A., Polo, T., \& Jiménez, J. (2010). Organización de la orientación universitaria en el Espacio Europeo de Educación Superior: La Unidad de Orientación de Centro. Electronic Journal of Research in Educational Psychology, 8(3), 1387-1404.

Villar-Varela, M., \& Méndez-Lois, M. (2014). Necesidades de orientación profesional de las mujeres gallegas en función de la edad. Revista Española de Orientación y Psicopedagogía, 25(2), 127-138. doi: 10.5944/reop.vol.25.num.2.2014.13524 


\section{Apéndice 1 \\ CUESTIONARIO DE NECESIDADES DE ORIENTACIÓN UNIVERSITARIA EN EL ECUADOR}

Paulina E. Moreno-Yaguana y María Fe Sánchez-García

\section{UTED}

Instrucciones: Marque una X en la respuesta o escriba en el espacio que lo solicite.

1. Sexo:

2. Edad: años

$\square$ Mujer $\square$ Hombre

3. Estado Civil:

$\square$ Soltero(a) $\square$ Casado(a) $\square$ Divorciado(a) $\quad$ Unión Libre $\square$ Viudo (a)

4. Carrera que cursa:

5. Nivel/Ciclo:

6. ¿Ud. presenta algún tipo de discapacidad? $\square \mathrm{Si} \quad \square$ No

7. En caso afirmativo señale el tipo de discapacidad:

$\square$ Auditiva $\square$ Física $\square$ Intelectual $\square$ Lenguaje $\square$ Psicológica $\square$ Visual

8. Porcentaje de discapacidad: $\%$

9. Posee carnet del CONADIS
$\square \mathrm{Si}$
$\square$ No

10. ¿Qué tipo de apoyo brinda la universidad en relación a su discapacidad?
$\square$ Económico
$\square$ Infraestructura (accesibilidad física)
$\square$ Académico
$\square$ Ninguno
$\square$ Psicológico
$\square$ Otros:
$\square$ Médico
Indicar:

Para realizar los estudios universitarios ¿Usted se trasladó de su lugar de residencia?

$\square \mathrm{Si} \quad \square$ No

En caso afirmativo, indique el lugar del cual se trasladó:

País: __ 13. Ciudad:

14. Parroquia: Cantón:

Además de realizar sus estudios. ¿Ud. trabaja?

$\square \mathrm{Si} \quad \square \mathrm{No}$

En caso afirmativo, indique:

16. Cuál es su ocupación?

17. ¿Cuántas horas semanales dedica a su trabajo?

18. Valore en qué grado ha necesitado orientación sobre los aspectos que le proponemos. Considere la siguiente escala: $0=$ ninguna necesidad $/ 5=$ máxima necesidad

\section{ASPECTOS ACADÉMICOS}

a-Orientación vocacional para la elección de su carrera.

b-Información sobre el sitio donde cursaré mis estudios

c-El proceso de matriculación.

d-Elegir correctamente las asignaturas

e-Acceder a cursos de nivelación académica.

f- La adquisición de hábitos de estudio.

g-El acompañamiento de un tutor(a) que le ayude a superar las dificultades académicas.

i-Obtener becas universitarias

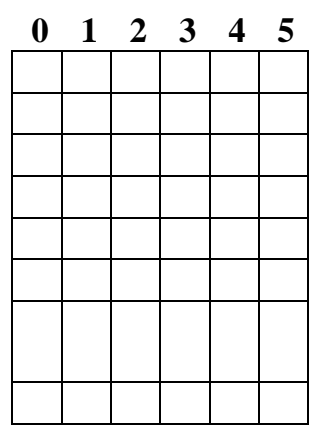

$\begin{array}{llllll}0 & 1 & 2 & 3 & 4 & 5\end{array}$ 


\section{ASPECTOS PROFESIONALES}

$\mathrm{k}$-La inserción laboral futura (campos laborales y empleo)

1-La planificación y el desarrollo de mi proyecto profesional y vital... m-Desarrollar habilidades para la búsqueda de empleo (CV, entrevista de selección, carta de presentación, etc.).

n-Adquirir habilidades de emprendimiento.

\section{ASPECTO PERSONALES}

o-Adaptación a la ciudad donde cursaré los estudios

p-Orientación para el autoconocimiento de mis cualidades y potencialidades personales.

q-Desarrollar mis habilidades sociales (de relación con el profesorado, compañeros, etc.).

r-El estrés y la depresión.

s-Trastornos de alimentación

$\mathrm{t}$-Orientación para mejorar la convivencia familiar.

u-Prevenir adicciones como: alcohol, drogas, juegos de azar, etc

v-Formación y orientación sobre relaciones afectivas y sexualidad
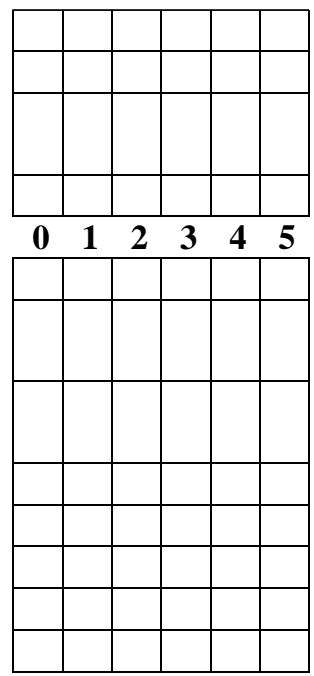

19. ¿Valore en qué grado considera necesario que su Universidad oferte servicios cómo...? Considere la siguiente escala: $0=$ ninguna necesidad $/ 5=$ máxima necesidad

a-Alojamiento universitario.

b-Programas de acogida a los estudiantes de nuevo ingreso.

c-Programas de atención a la diversidad de estudiantes.

d-Programas de apoyo al rendimiento académico.

e-Atención médica...

f-Unidad de apoyo psicológico.

g-Asesoramiento ocupacional y bolsa de empleo.

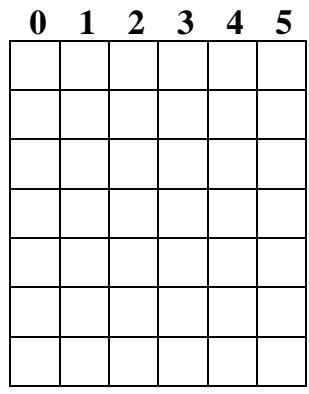

20. En este espacio, puede proponer aquello que entiende que debe proporcionar un servicio de orientación en la universidad o cualquier otra sugerencia u opinión:

\section{GRACIAS}

